\section{artigo original}

\author{
Carlos A. da Silva \\ Walter C. de Lima
}

Departamento de Clínica Médica, Faculdade de Ciências Médicas, Universidade Estadual de Campinas, SP.

Recebido em 06/09/01

Revisado em 08/03/02 e em 12/07/02

Aceito em 12/08/02

\title{
Efeito Benéfico do Exercício Físico no Controle Metabólico do Diabetes Mellitus Tipo 2 à Curto Prazo
}

\section{RESUMO}

O objetivo deste trabalho foi analisar o efeito do exercício físico regular no controle glicêmico em indivíduos diabéticos tipo 2, tratados e nãotratados com insulina, em pessoas da região do Vale do Itajaí, SC, com idades entre 45 e 75 anos. Foram realizados testes de glicemia jejum (GJ), hemoglobina glicosilada (HbAl) e glicemia capilar nos clientes da Unicardio/HSC e nos participantes da Associação dos Diabéticos de Blumenau $(n=33)$, onde estes passaram por um programa de exercício físico de 10 semanas, após as quais os participantes da amostra foram reavaliados. Foi avaliado também: perfil lipídico, pressão arterial, freqüência cardíaca de repouso e índice de massa corporal. Os instrumentos utilizados foram: o exame de sangue em jejum para avaliação laboratorial e a glicemia capilar. Para análise estatística utilizou-se o teste " $t$ " de Student e a reta ajustada de mínimos quadrados, pela regressão linear. Obteve-se os seguintes resultados: glicemia capilar média pré-teste $=179 \mathrm{mg} / \mathrm{dL}$ e pós-teste $=148 \mathrm{mg} / \mathrm{dL} ; \mathrm{HbAl}$ média préteste $=9,5 \%$ e pós-teste = 8,5\%; GJ média pré-teste $=164,8 \mathrm{mg} / \mathrm{dL}$ e pósteste $=156,4 \mathrm{mg} / \mathrm{dL}$. Estes resultados permitem concluir que o exercício físico é de grande importância no controle glicêmico do indivíduo diabético tipo 2, tratado ou não-tratado com insulina, diminuindo a glicemia e a HbAl. (Arq Bras Endocrinol Metab 2002;46/5:550-556)

Descritores: Exercício físico; Glicemia; Hemoglobina glicosilada; Diabetes mellitus

\begin{abstract}
Beneficial Effect of Short Time Physical Exercise on the Metabolic Control of Type 2 Diabetes Mellitus.

The objective of our study was to analyze the effect of physical exercise on the control of glycemia in insulin treated and non-treated type 2 diabetic subjects ( 45 to 75 years) from Vale do Itajaí, SC. Fasting glycemia (FG), glycated hemoglobin ( $\mathrm{HbAl}$ ) and capillary glycemia were performed in clients of Unicardio/HSC and in participants of the Blumenau Diabetes Association ( $n=33$ ), before and after a 10 week-program of physical exercise, considering that all were re-submitted to the same evaluation after the program. The evaluation also included: lipid profile, blood pressure, heart rate and body mass index (BMI). The instruments were: FG test for laboratory evaluation and capillary glycemia. Statistical analysis used Student's " $t$ " test and the adjusted straight line of square minima, for linear regression. The following results were obtained: average pre- and post test capillary glycemia $=179 \mathrm{mg} / \mathrm{dL}$ and $148 \mathrm{mg} / \mathrm{dL}$, respectively; pre- and post test $\mathrm{HbA} 1=9.5 \%$ and $8.5 \%$; pre- and post test $\mathrm{FG}=164.7 \mathrm{mg} / \mathrm{dL}$ and $156.4 \mathrm{mg} / \mathrm{dL}$. We conclude that physical exercise is important for glycemic control of insulin treated and non-treated type 2 diabetic subjects, reducing both the glucose levels and $\mathrm{HbA}$. (Arq Bras Endocrinol Metab 2002;46/5:550-556)
\end{abstract}

Keywords: Physical exercise; Glicemia; Glycated hemoglobin; Diabetes mellitus 
A RAPIDEZ E A EXTENSÃo DA URBANIZAÇÃo são algumas das características do século XX. Esse processo provocou modificações agressivas nos hábitos dietéticos e no estilo de vida das pessoas, acarretando enorme redução nos níveis de atividade física. Essas mudanças provocaram um significativo impacto sobre a saúde e a mortalidade de grandes populações, e constitui-se em um grave problema de saúde pública (1). Por isso, o perfil das doenças sofreu profundas mudanças em nosso meio, observando-se uma alteração da mortalidade decorrente de doenças infecto-contagiosas e materno-infantis, pela mortalidade advinda de causas externas e disfunções classificadas como de origem crônico-degenerativas (2). A crescente incidência do diabetes mellitus (DM) na população mundial, como a prevalência dos EUA chegando a 10 milhões de pessoas com DM tipo 2 (DM2) constituindo $5 \%$ da população americana, é uma das conseqüências mais graves dessas modificações (2).

O DM é um dos mais importantes problemas de saúde mundial, tanto em número de pessoas afetadas como de incapacitação e de mortalidade prematura, bem como dos custos envolvidos no seu tratamento. Há uma tendência ao aumento de sua prevalência, estimando-se que o DM na população brasileira esteja em $7 \%$, sendo que somente em São Paulo esse número chega a $9 \%$ na faixa etária dos 30 aos 59 anos e, na faixa etária dos 60 aos 69 anos chega a 13,4\% (3). Entre os tipos de diabetes, o DM2 é o de maior incidência, alcançando entre 90 e $95 \%$ dos casos, acometendo geralmente indivíduos de meia idade ou em idade avançada, podendo uma hiperglicemia estar presente por vários anos, anteriormente ao seu diagnóstico (4). O tratamento do DM envolve o uso de antidiabéticos orais e/ou insulina, dieta e atividade física.

Estudos apoiam a evidência de que o exercício físico reduz os níveis de glicemia em diabéticos (512). Porém, outros estudos não demonstraram esse benefício de forma conclusiva (13-17). Frente a esses dados, nosso objetivo foi estudar o efeito do exercício físico regular no controle glicêmico em indivíduos com DM2.

\section{MATERIAL E MÉTODOS}

Esta é uma pesquisa do tipo experimental, da qual foram retirados da população de diabéticos da Clínica Unicardio/HSC de Blumenau e da Associação dos Diabéticos de Blumenau uma amostra de 33 sujeitos, conforme seleção pelo método probabilístico com a técnica casual simples, cujo critério de inclusão foi ser sedentário. Todos os sujeitos da amostra tinham DM2, sendo $8(24,2 \%)$ tratados e $25(75,8 \%)$ nãotratados com insulina; com idades entre 45 e 75 anos, sendo 18 homens e 15 mulheres; $11(33,3 \%)$ sujeitos faziam tratamento com antidiabético oral e $22(66,6 \%)$ não faziam esse tipo de tratamento; $21(63,6 \%)$ sujeitos tinham no máximo 2 anos de diagnóstico, enquanto 12 tinham mais de 2 anos de diagnóstico; 7 (21\%) já tinham cardiopatia; 5 homens e 9 mulheres estavam com IMC (índice de massa corporal) acima do valor limite $\left(25 \mathrm{~kg} / \mathrm{m}^{2}\right) ; 17(5 \mathrm{l}, 5 \%)$ sujeitos da amostra estavam com a pressão arterial elevada, acima de 139/89mmHg. Foram critérios de exclusão: 1) O indivíduo que teve seu tratamento modificado durante o programa de exercícios (antidiabéticos orais e/ou insulina); 2) Não seguiu dieta conforme o inquérito alimentar inicial; 3) Faltou ao programa de exercícios físicos. Todos os participantes assinaram o termo de consentimento previamente aprovado pelo Comitê de Ética em pesquisa da Universidade do Estado de Santa Catarina - UDESC.

Utilizou-se um modelo de delineamento experimental de pré-teste e pós-teste aplicado ao grupo. Foram medidas e analisadas as variáveis: glicemia (glicemia jejum medida pré-teste e pós-teste, glicemia capilar, calculada a média das medidas em três dias alternados de exercício físico antes e em jejum e depois do exercício físico, hemoglobina glicosilada ( $\mathrm{HbAl})$ medida pré-teste e pós-teste); lipídios plasmáticos (CTOTAL-colesterol total; LDL-colesterol (LDL-C); HDL-colesterol (HDL-C); triglicerídeos (TRIG), medidos pré-teste e pós-teste; freqüência cardíaca de repouso medida em todas as sessões de exercício físico; pressão arterial medida em todas as sessões de exercício físico; e massa gorda medida pré-teste e pós-teste. Para coleta de dados, foram feitas duas rotinas: a rotina de laboratório e a rotina de campo. A rotina de laboratório, por meio de coleta de sangue em jejum: para medir a glicemia em jejum (método reativo seco vitrus para glicose); hemoglobina glicosilada (método Abbott $\operatorname{Imx}$ ); colesterol total (método retivo seco para colesterol); HDL colesterol (método análise de colesterol com kit vitros para HDL-C); triglicerídeos (método retivo seco para TRIG); e LDL colesterol (calculado utilizando a fórmula LDL-C $=(\mathrm{C}-$ Total - HDL-C $)-$ TRIG. A rotina de campo: para coleta de dados referentes a fármacos anotados em formulário próprio; anamnese nutricional por um inquérito alimentar avaliado pelo Sistema Sapaf Adulto 3.0 pré-teste e pós-teste; ficha de identificação e diagnóstico para enquadrar os sujeitos na pesquisa e avaliar a condição sócio-econômica e queixas pela percepção subjetiva; massa corpórea medida pela balança 
(marca Plenna) e estatura medida pelo estadiômetro (marca Soehnle), para cálculo do IMC pela fórmula de Quetelet (massa corporal expressa em quilograma dividido pelo quadrado da estatura em metros); freqüência cardíaca medida no pulso antes do exercício, no meio e no final; pressão arterial medida antes e após exercício; e glicemia capilar (Medisense Precision QID).

Como tratamento experimental, foi feito um programa de exercícios físicos, com 10 semanas de duração, sendo 4 sessões por semana de 60 minutos cada. Cada sessão estava assim dividida: 5 minutos de aquecimento (exercícios de alongamento e de circundunção de membros e tronco); 40 minutos de exercícios aeróbios (caminhada, corrida ou bicicleta); 10 minutos de exercícios de resistência muscular localizada (com pesos); e 5 minutos de resfriamento (com exercícios de alongamento, flexibilidade e relaxamento). Os exercícios obedeceram uma intensidade de leve a moderada ( $50 \%$ a $80 \%$ da Fcmáx.) (18), calculada pela fórmula de Karvonen (Fcmáx. = 220 - idade) $(19-21)$.

Foram controladas as variáveis: ingesta de alimentos, por meio de uma anamnese nutricional e calculada no programa Sapaf Adulto 3.0, fazendo-os permanecer nesta até o final do programa de exercícios físicos (22); e fármacos, registrando seus medicamentos no início do programa de exercícios físicos.

Foi realizado como procedimento estatístico, para comparação das médias o teste " $\mathrm{t}$ " de Student, com nível de significância a $5 \%(\mathrm{p} \leq 0,05)$, e para uma análise de tendência para série temporal das medidas a reta ajustada de mínimos quadrados (regressão linear).

\section{RESULTADOS}

A diminuição crônica da glicemia, conforme visto nos gráficos 1 e 2 , demonstra ser significativa para $\mathrm{p}<0,05$ por um programa de exercício físico regular. $\mathrm{O}$ indivíduo com DM2 tem sua glicemia diminuída pelo efeito agudo do exercício físico, mostrado na glicemia capilar, a qual foi coletada antes e após uma sessão de exercício.

O gráfico 3 apresenta as médias pré-teste e pósteste dos lipídios plasmáticos, e verifica-se que o grupo estudado apresenta níveis de C-TOTAL, LDL-C e TRIG menores, e o HDL-C maior $(\mathrm{p}<0,05)$.

Quanto à freqüência cardíaca de repouso, foram encontradas diferenças altamente significativas, conforme gráfico 4 . No gráfico 5 , a reta ajustada à média, demonstra uma forte correlação $(r=-0,99)$ inversamente proporcional à progressão do programa de exercício físico com a frequiência cardíaca de repouso.

$\mathrm{Na}$ pressão arterial, verificou-se que o grupo não apresentou diferença significativa após o programa de exercício físico. Observou-se uma fraca correlação entre exercício físico e melhora da pressão arterial, tanto na PAS $(r=-0,45)$ quanto na PAD $(r=-0,30)$.

$\mathrm{O}$ efeito do programa de exercício físico no IMC foi significativo, fazendo ocorrer uma discreta diminuição de $0,65 \mathrm{Kg} / \mathrm{m}^{2}$ na média do grupo, visto no gráfico 6 , analisado estatisticamente pelo teste $t$ de Student (teste $\mathrm{t}=2,48 ; \mathrm{t}$ crítico $=2,04 ;$ probabilidade $\mathrm{P}=0,0185$; nível de significância $\mathrm{a}=0,05$ ).

Com relação à hipoglicemia, não houve ocorrência em nenhum sujeito da pesquisa durante o programa

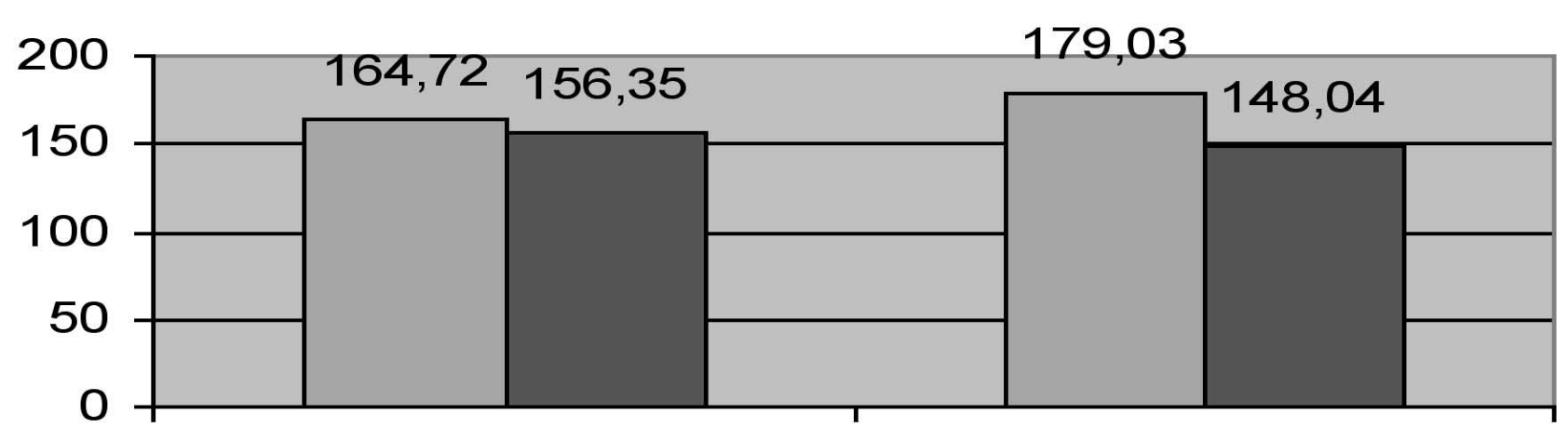

Glic. Jejum

Glic. Capilar

\section{$\square$ Pré-teste $\square$ Pós-teste}

Gráfico 1. Médias pré-teste e pós-teste da glicemia jejum e da glicemia capilar do grupo. 


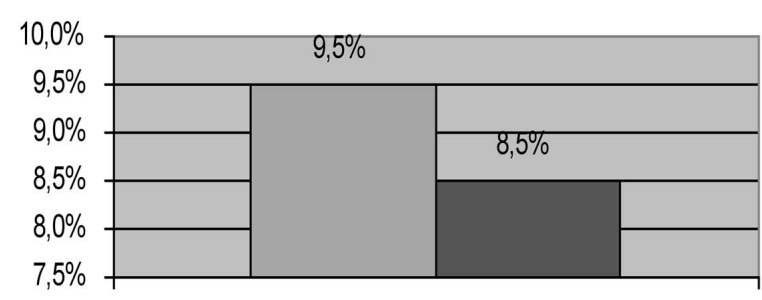

Hem. Glicos.

$\square$ Pré-teste $\square$ Pós-teste

Gráfico 2. Médias pré-teste e pós-teste da hemoglobina glicosilada do grupo.

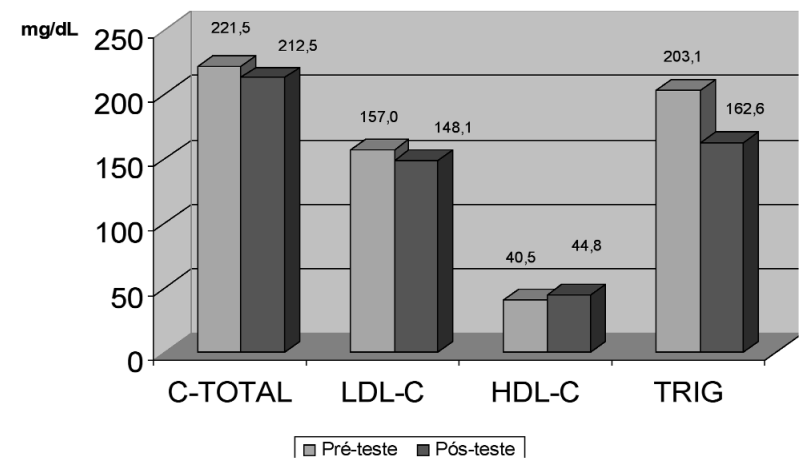

Gráfico 4. Médias pré-teste e pós-teste da freqüência cardíaca de repouso do grupo.

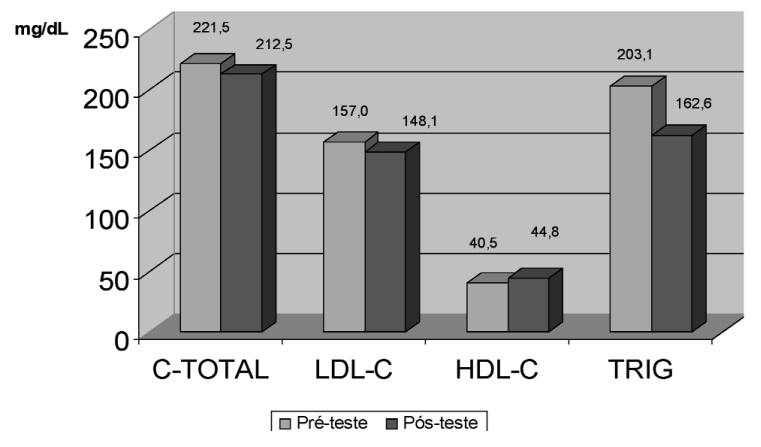

Gráfico 3. Médias pré-teste e pós-teste dos lipídios plasmáticos (C-TOTAL, LDL-C, HDL-C e TRIG) do grupo.

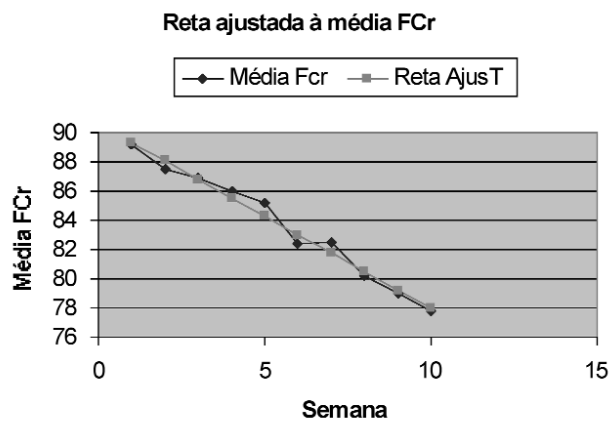

Gráfico 5. Reta ajustada da freqüência cardíaca de repouso.

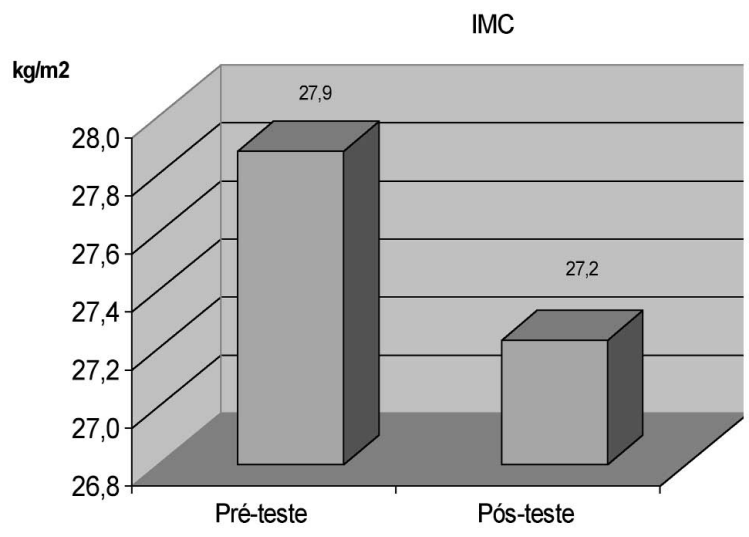

Gráfico 6. Médias pré-teste e pós-teste do índice de massa corporal do grupo. 
de exercício físico. A temperatura ambiente no período da pesquisa se estabeleceu com uma média de $31^{\circ} \mathrm{C}$, nos meses de janeiro a abril de 2001, aferindo uma mínima de $23^{\circ} \mathrm{C}$ e uma máxima de $39^{\circ} \mathrm{C}$. Quanto à ingestão de alimentos, $69,7 \%$ do sujeitos se mantiveram dentro da análise alimentar feita antes do início do programa de exercício físico, estabelecendo uma média diária de consumo de nutrientes de proteínas $=9,3 \%$; carboidratos $=$ $56,1 \%$; e lipídios $=34,6 \%$. Quanto à condição sócioeconômica, $78,7 \%$ dos sujeitos tinha uma renda mensal acima de R\$ 1.000,00, condição considerada muito boa. Quanto às complicações cardiovasculares, 21,2\% dos sujeitos já tinham diagnóstico de doença cardiovascular aterosclerótica antes da pesquisa. Após a intervenção houve melhora nos níveis de glicemia, TRIG, HDL-C e IMC. Os sujeitos da pesquisa relataram subjetiva melhora em algumas queixas após o início do programa, como depressão; sono (insônia); dores nos membros inferiores; maior sensação de bem estar; e melhor relacionamento social.

\section{DISCUSSÃO}

No presente estudo, o programa de exercício físico em indivíduos com DM2 induziu melhora nas variáveis: glicemia de jejum, $\mathrm{HbAl}$, lipídios plasmáticos, freqüência cardíaca de repouso e índice de massa corporal. Os resultados obtidos para o grupo de diabéticos tipo 2 , estudado após 10 semanas desse programa, estão de acordo com o esperado para o tratamento dessa doença.

A glicemia de jejum isolada após o treinamento físico baixou. Isso poderia ser justificado pelo efeito benéfico do exercício, tal como a melhora da captação de glicose que se encontra aumentada durante o exercício físico, mesmo com baixos níveis insulinêmicos (23). Pratley e cols. (24), estudando pessoas com mais de 65 anos de idade fazendo exercícios físicos aeróbios durante 9 meses, demonstrou que esse tipo de treinamento diminui significativamente as concentrações de insulina estimuladas pela glicose. Zinker e cols. (25) em sua pesquisa com três grupos de indivíduos diabéticos, onde o primeiro grupo fez exercícios físicos, o segundo grupo usou metformina, e o terceiro grupo usou troglitazone, identificou que o grupo que mais melhorou a sensibilidade à insulina foi o que fez exercícios físicos. Outros autores $(7,10,26,27)$ evidenciam que o efeito do exercício físico agudo em indivíduos diabéticos tipo 2 consiste num notável aumento na utilização de glicose se comparado com indivíduos diabéticos tipo 2 não treinados.

Importante considerar que os indivíduos com DM2, tratados ou não com insulina, tiveram o mesmo efeito hipoglicemiante como resposta do exercício físico.
Portanto, fica clara a importância do exercício físico para o indivíduo com DM 2, tratado ou não com insulina, como resposta do seu efeito agudo (efeito de uma sessão de exercício físico). Silva e cols. (12) salientam a importância do exercício físico diário, facilitando o controle do diabetes. Esses exercícios devem ser executados com uma intensidade de leve a moderado ( $50 \%$ a $80 \%$ da Fcmáx., progressivamente), com exercícios aeróbios (caminhada, corrida, bicicleta) e exercícios com peso que desenvolvam a resistência muscular localizada (até 30\% de carga), com uma duração de mais ou menos 60 minutos, de maneira regular.

Os níveis de lipídios plasmáticos foram melhorados após as 10 semanas do programa de exercício físico. Alguns estudos $(7,8,11,28,29)$ demonstram que a reposta ao exercício físico melhora os níveis de C-TOTAL, HDL-C, LDL-C e TRIG. Destaca-se o importante papel do exercício físico na diminuição dos triglicerídeos e no aumento do HDL colesterol.

No presente estudo percebeu-se uma melhora da eficiência cardíaca observada pela redução em mais ou menos $10 \mathrm{bpm}$ da freqüência cardíaca de repouso. Pollock e cols. (30) formaram 2 grupos na faixa etária de 40 a 50 anos de idade, em que um grupo fez exercícios físicos durante 6 meses e o outro grupo não fez exercícios físicos, e perceberam uma melhora média de 13\% na freqüência cardíaca de repouso do grupo que fez exercícios físicos, mais ou menos $10 \mathrm{bpm}$, os quais são dados concordantes com os nossos.

$\mathrm{O}$ grupo pesquisado não apresentou melhora na pressão arterial após as 10 semanas de exercícios físicos. Martins e cols. (31) também não encontraram alteração significativa do exercício físico sobre a pressão arterial em indivíduos diabéticos hipertensos.

O IMC do grupo estudado foi melhorado, observado pela queda em seus valores pós-treinamento físico. Pratley e cols. (24) evidenciaram que o treinamento com exercícios físicos aeróbios diminui a quantidade de gordura corporal em indivíduos idosos, e isso pode mediar alguns efeitos metabólicos do exercício físico aeróbio, principalmente pelo excesso da gordura abdominal estar associada com a resistência à insulina e hiperinsulinemia. Nossos dados são concordantes com outros estudos $(7,8,10,25,27,28,32)$, que também observaram a diminuição do IMC após um programa de exercício físico.

\section{CONCLUSŌES}

Um programa de exercício físico regular, de intensidade moderada, auxilia no controle glicêmico do 
indivíduo com DM2, tratado ou não com insulina, sendo que seu efeito já é observado em uma sessão de exercício.

Fica caracterizado que um programa de exercício físico bem orientado e regular melhora os níveis de lipídios plasmáticos, principalmente diminuindo significativamente os triglicerídeos e aumentando o HDL$\mathrm{C}$, mas sem alteração significativa no C-TOTAL e o LDL-C.

O exercício físico regular melhora a eficiência cardíaca, diminuindo a freqüência cardíaca de repouso em até $10 \mathrm{bpm}$, efeito evidenciado à partir de 10 semanas de programa.

Não ficou comprovado neste trabalho o efeito crônico do exercício físico na pressão arterial, cabendo ser necessário mais pesquisa nesta variável.

Nesta pesquisa, ocorreram alterações significativas no IMC dos indivíduos com DM2.

Portanto, conclui-se que é uma conduta bem recomendada, um programa de exercício físico, com atividades aeróbias e de resistência muscular localizada, 4 vezes por semana, com sessões de 60 minutos para DM2, resultando nos seguintes benefícios: melhora na glicemia de jejum e $\mathrm{HbAl}$; diminuição de triglicerídeos e aumento de HDL-C; diminuição da freqüência cardíaca de repouso, melhorando a eficiência cardíaca e auxiliando na diminuição do IMC.

\section{REFERÊNCIAS}

1. Silveira Netto E. Atividade física para diabéticos. $1^{\mathrm{TM}}$ ed. Rio de janeiro: Sprint, 2000.

2. Duncan $\mathrm{BB}_{\text {,., }}$ et al. Altos coeficientes de mortalidade em populações adultas brasileiras: uma comparação internacional. Rev Assoc Bras 1992;38(3):138-44.

3. Oliveira EF, Granja LA, Wajchenberg BL. Cardiopatia no diabético. Rev Bras Cardiol 2000;2(3):103-15.

4. Martins DM. Exercício físico no controle do diabetes mellitus. $1^{\text {TM }}$ ed. Guarulhos: Phorte, 2000, p.3-14.

5. Peyrot M, Rubin RR. Modeling the effect of diabetes education on glycemic control. Diabetes Educator 1994;20(2): 143-8.

6. Raz I, Hauser E, Bursztyn M. Moderate exercise improves glucose metabolism in uncontrolled elderly patients with noninsulin-dependent diabetes mellitus. Isr J Med Sci 1994;30(10):766-70.

7. Leong KS, Wilding JP. Obesity and diabetes. Bailliére's Clin Endocrinol Metab 1999;13(2):221-37.

8. Kannan V. Diet, activity and diabetes. J Assoc Phys India 1999:47(8):761-3.

9. Glasgow R, Fisher EB, Anderson BJ, Lagrega A, Marrero $\mathrm{D}$, Johnson $\mathrm{SB}_{\text {, }}$ et al. Behavioral science in diabetes. Diabetes Care 1999:22(5):832-3.
10. Gumbiner B. The treatment of obesity in type 2 diabetes mellitus. Primary Care 1999;26(4):869-83.

11. Rigla M, Sánchez-Quesada JL, Ordóñez-Lianos J, Prat T, Caixàs $A$, Jorba $O$, et al. Effect of physical exercise on lipoprotein (a) and low-density lipoprotein modifications in type-1 and type-2 diabetic patients. Metabolism 2000;49(5):640-7.

12. Silva CA, Lima WC. O exercício físico e o paciente diabético tipo II. Dynamis 2001;9(34):49-60.

13. Abe R, Fujinuma $H$. Exercise in elderly NIDDM. NipponRonen-Igakkaiz-Zasshi 1993;30(4):283-7.

14. Bell DS. Exercise for patients with diabetes: benefits, risks, precautions. Postgraduate Medicine 1992; 92(1): 183-98.

15. Blonck MC, Jacobs MA, Biesheuvel EH, Weeda-Mannak WL, Heine RJ. Influences on weight loss in type 2 diabetic patients: little long-term benefit from group behaviour therapy and exercise training. Diab Med 1994; 11 (5):449-57.

16. Jun JY. The effects of programmed jogging on metabolism and cardio-pulmonary function of type II diabetic patients. Kanhoha Tambu 1994;3(1):19-42.

17. Selam JL, Casassus P, Bruzzo F, Leroy C, Slama G. Exercise is not associated with better diabetes control in type 1 and type 2 diabetic subjects. Acta Diabetol 1992;29(1):11-3.

18. ACSM - American College of Sports Medicine. Research manual for guidelines for exercise testing and prevention. $2^{\text {nd }}$ ed. Philadelphia: Lea and Febiger, 1993.

19. Fox EL, Bowers RW, Foss ML. Bases fisiológicas da educação física e dos desportos. $4^{a}$ ed. Rio de Janeiro: Guanabara Koogan, 1991.

20. Edwards S. O livro do monitor de freqüência cardíaca. $1^{\text {TM }}$ ed. Finland: Polar Electro Oy, 1994.

21. Brick M. Multi-esporte de precisão. $1^{\mathrm{TM}}$ ed. Finland: Polar Electro Oy, 1995.

22. Guedes DP. Sapaf Adulto 3.0: Sistema de avaliação e prescrição de atividade física: manual do usuário. $1^{\text {TM }}$ ed. Londrina: Midiograf, 1996.

23. Luciano E, Bessa Lima F. Metabolismo de ratos diabéticos treinados submetidos ao jejum e ao exercício agudo. Rev Cienc Biomed 1997; 18:47-60.

24. Pratley RE, Hagberg JM, Dengel DR, Rogus EM, Muller DC, Goldberg AP. Aerobic exercise training induced reductions in abdominal fat and glucose stimulated insulin responses in mild-aged and older men. J Am Ger Soc 2000;48(9):2022-33.

25. Zinker BA. Nutrition and exercise in individuals with diabetes. Clin Sports Med 1999; 10(3):585-606.

26. Dela F, Mikines KJ, Larsen JJ, Galbo H. Glucose clearence in aged trained skeletal muscle during maximal insulin with superimposed exercise. J App Phys 1999;87(6):2059-67.

27. Hickner AC, Racette SB, Binder EF, Fisher JS, Kohrt WM. Supression of whole body and regional lipolysis by insulin: effects of obesity and exercise. J Clin Endocrinol Metab 1999;84(11):217-27. 
28. Wheeler ML. Nutrition management and physical activity as treatments for diabetes. Primary Care 1999;26(4):857-68.

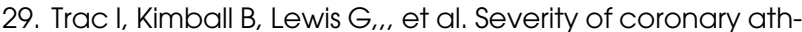
erosclerosis in type II diabtes mellitus is related to the number of circulating, triglyceride-rich lipopropein particles. Aterioscl Thromb Vasc Biol 1997; 17:3633-8.

30. Pollock ML, Wilmore JH. Exercício na saúde e na doença: avaliação e prescrição para prevenção e reabilitação. $2^{\mathrm{TM}^{3}}$ ed. Rio de Janeiro: Medsi, 1993.

31. Martins DM, Duarte MFS. Efeito do exercício físico sobre o comportamento da glicemia em indivíduos diabéticos. Rev Bras Ativ Fís Saude 1998;3(3):32-44.
32. Dipietro L, Seeman TE, Stachenfeld NS, Katz LD, Nadel ER. Moderate-intensity aerobic training improves glucose tolerance in aging independent of abdominal adiposity. J Am Ger Soc 1998;46(7):867-78.

\section{Endereço para Correspondência}

Carlos Alberto da Silva

Rua Natal, 30 - apto 102

89107-000 Pomerode, SC

e.mail: carlosas.bnu@terra.com.br 